\title{
Erratum: Holographic RG flows in six dimensional $F(4)$ gauged supergravity
}

\section{Parinya Karndumri}

String Theory and Supergravity Group, Department of Physics,

Faculty of Science, Chulalongkorn University,

254 Phayathai Road, Pathumwan, Bangkok 10330, Thailand

Thailand Center of Excellence in Physics, CHE, Ministry of Education,

319 Dusit, Bangkok 10400, Thailand

E-mail: parinya.ka@hotmail.com

ERRATUM TO: JHEP01(2013)134

ArXiv EPRINT: 1210.8064

An argument made in the original paper is not correct. The critical point reported in equation (3.3) is actually supersymmetric. Furthermore, the fermionic supersymmetry transformations given in equations (2.9), (2.10) and (2.11) contain some typos. The correct equations are

$$
\begin{aligned}
\delta \psi_{\mu A}= & D_{\mu} \epsilon_{A}-\frac{1}{24}\left[A e^{\sigma}+6 m e^{-3 \sigma}\left(L^{-1}\right)_{00}\right] \epsilon_{A B} \gamma_{\mu} \epsilon^{B} \\
& -\frac{1}{8}\left[B_{t} e^{\sigma}-2 m e^{-3 \sigma}\left(L^{-1}\right)_{t 0}\right] \gamma^{7} \sigma_{A B}^{t} \gamma_{\mu} \epsilon^{B}, \\
\delta \chi_{A}= & \frac{1}{2} \gamma^{\mu} \partial_{\mu} \sigma \epsilon_{A B} \epsilon^{B}+\frac{1}{24}\left[A e^{\sigma}-18 m e^{-3 \sigma}\left(L^{-1}\right)_{00}\right] \epsilon_{A B} \epsilon^{B} \\
& -\frac{1}{8}\left[B_{t} e^{\sigma}+6 m e^{-3 \sigma}\left(L^{-1}\right)_{t 0}\right] \gamma^{7} \sigma_{A B}^{t} \epsilon^{B}, \\
\delta \lambda_{A}^{I}= & P_{r i}^{I} \gamma^{\mu} \partial_{\mu} \phi^{i} \sigma_{A B}^{r} \epsilon^{B}+P_{0 i}^{I} \gamma^{7} \gamma^{\mu} \partial_{\mu} \phi^{i} \epsilon_{A B} \epsilon^{B}-\left(2 i \gamma^{7} D^{I}{ }_{t}+C^{I}{ }_{t}\right) e^{\sigma} \sigma_{A B}^{t} \epsilon^{B} \\
& +2 m e^{-3 \sigma}\left(L^{-1}\right)^{I}{ }_{0} \gamma^{7} \epsilon_{A B} \epsilon^{B} .
\end{aligned}
$$

Using the scalar parametrization given in equation (3.1) and the metric ansatz (4.4) as well as the projection $\gamma_{r} \epsilon=\epsilon$, we find the corresponding BPS equations

$$
\begin{aligned}
b^{\prime} & =-\frac{1}{4} e^{a-3 b}\left(e^{4 b}-1\right)\left[g_{1}\left(1+e^{2 b}\right)-g_{2}\left(e^{2 b}-1\right)\right], \\
a^{\prime} & =-\frac{1}{16} e^{a-3 b}\left[g_{1}\left(1+e^{2 b}\right)^{3}-g_{2}\left(e^{2 b}-1\right)^{3}\right]+\frac{3}{2} m e^{-3 a}, \\
A^{\prime} & =\frac{1}{16} e^{a-3 b}\left[g_{1}\left(1+e^{2 b}\right)^{3}-g_{2}\left(e^{2 b}-1\right)^{3}\right]+\frac{1}{2} m e^{-3 a} .
\end{aligned}
$$


After correcting the typos in the critical point (3.3) namely the values of $a$ and the $A d S_{6}$ radius are given, respectively, by

$$
a=\frac{1}{4} \ln \left[-\frac{3 m \sqrt{g_{2}^{2}-g_{1}^{2}}}{g_{1} g_{2}}\right] \quad \text { and } \quad L=\frac{1}{2 m}\left[-\frac{3 m \sqrt{g_{2}^{2}-g_{1}^{2}}}{g_{1} g_{2}}\right]^{\frac{3}{4}},
$$

we find that the critical point (3.3) is actually a critical point of the potential (3.2) and satisfies all of the above BPS equations with the corrected $A d S_{6}$ radius. This critical point can also be seen from the related BPS equations studied in [1].

Finally, the RG flow from the $\mathrm{SU}(2)_{R} \times \mathrm{SU}(2)$ CFT in the UV to this critical point is driven by a vacuum expectation value of an operator of dimension 3 . The flow solution can also be obtained from the BPS equations (4), (5) and (6). On the other hand, the mass spectrum in the original paper is correct as it stands.

Open Access. This article is distributed under the terms of the Creative Commons Attribution License (CC-BY 4.0), which permits any use, distribution and reproduction in any medium, provided the original author(s) and source are credited.

\section{References}

[1] P. Karndumri, Gravity duals of $5 D N=2 S Y M$ theory from $F(4)$ gauged supergravity, Phys. Rev. D 90 (2014) 086009 [arXiv: 1403.1150] [INSPIRE]. 